\title{
Instructional Crisis Communication: Connecting Ethnicity and Sex in the Assessment of Receiver-Oriented Message Effectiveness
}

\author{
Robert S. Littlefield ${ }^{1}$, Kimberly Beauchamp ${ }^{1}$, Derek Lane ${ }^{2}$, Deanna D. Sellnow ${ }^{2}$, Timothy L. Sellnow ${ }^{2}$, Steven \\ Venette $^{3} \&$ Bethany Wilson ${ }^{2}$ \\ ${ }^{1}$ North Dakota State University, United States \\ ${ }^{2}$ University of Kentucky, United States \\ ${ }^{3}$ University of Southern Mississippi, United States \\ Correspondence: Robert S. Littlefield, Professor of Communication, Fargo Chamber of Commerce NDSU \\ Distinguished Professor, North Dakota State University, United States. E-mail: R.Littlefield@ndsu.edu
}

Received: June 26, 2014

doi:10.5430/jms.v5n3p16
Accepted: July 14, 2014

Online Published: August 10, 2014

URL: http://dx.doi.org/10.5430/jms.v5n3p16

\begin{abstract}
This study explored the responses of receivers of risk messages that included all elements of the Internalization-Distribution-Explanation-Action (IDEA) learning cycle model to determine if culture, sex, and socio-economic status had any impact on receptivity and behavioral intention. Using a pre- and post-measures experimental design, 746 participants from different geographic areas within the United States watched prepared news clips. Participants identified their learning styles, the perceived message effectiveness, and their behavioral intentions following their observation. Results suggest that messages addressing all elements of the IDEA model were perceived as more effective by participants. Ethnicity and sex of participants in some cases made a difference regarding perception of message effectiveness.
\end{abstract}

Keywords: risk communication, message effectiveness, learning styles, behavioral intentions, ethnicity

\section{Introduction}

We know that effective crisis communication is needed to mitigate harm, manage reputations, and prepare and potentially reduce the likelihood of future incidents (Sellnow, Ulmer, Seeger, \& Littlefield, 2009). Despite the need to address multiple perspectives when developing crisis messages, organizational decision-makers often focus on the protection of their own reputations and minimizing the consequences of their actions (Seeger, Sellnow, \& Ulmer, 2003). Considerable scholarship has focused on this top-down perspective when studying the management of crisis, despite the fact that these sender-focused best practices are predicated on the inherent assumption that the publics will respond as directed during a crisis, which is not often the case (e.g., Hurricane Katrina). Kreps, Alibek, Bailey et al. (2005) suggested that "one of the biggest challenges" to designing effective crisis messages is "making relevant information accessible and understandable to highly varied subgroups in society" (p. 196).

Scholars have suggested that cultural traits and preferences may be one reason why messages are not received and acted upon by members of different cultural groups within the United States (e.g., Andrulis \& Brach, 2007; Sellnow et al, 2009; Littlefield, 2013). For example, some groups may prefer particular spokespeople, some may prefer certain types of messages based upon their sex, ethnicity, or learning style. In an effort to understand better how to make risk and crisis messages more accessible and effective, the present study explores the responses of the general public and several domestic minority ethnic groups within the United States to tailored risk and crisis messages. D. Sellnow, Lane, Littlefield, et al. (2013) studied the general public and found that tailoring messages based upon learning style preference, sex, and group type maximized their persuasive impact. They concluded: "instructions for self-protection [focusing on receiver-oriented strategies] will increase comprehension, garner desired response, and produce appropriate self-protective action" (p. 15). This study is an extension of that work currently being conducted by scholars at universities associated with the National Center for Food Protection and Defense, a Center of Excellence sponsored by the U. S. Department of Homeland Security. 


\section{Conceptual Framework}

\subsection{Impact of Culture on Message Reception}

Scholars have recognized the diversity of multiple publics and identified a number of factors affecting how culturally diverse groups respond to risk and crisis messages (Lindell \& Perry, 2004; Seeger et al., 2003; Sellnow et al., 2009). While varying from macro to micro in scope, these factors shape the way crisis messages are received. Sarbaugh (1979) organized these factors into four categories: code systems, perceptions about relationships and intent, knowledge and acceptance of normative beliefs and values, and worldviews.

Code system refers to verbal and nonverbal communication used by the various publics. Perceived relationships and intent corresponds with how spokespersons and publics respond to power, social connectedness, uncertainty, and assigned roles. Knowledge and acceptance of normative beliefs and values relates to the receptiveness of those in authority to those considered as minority, outsider, or vulnerable publics. Finally, worldview pertains to how people respond to the world around them. Perceptions about the nature of life, purpose of life, and relationship of humans to the afterlife affect how messages will be received.

Spence, Lachlan, and Griffin (2007) found that economic, social, political, and cultural factors influenced how some publics responded to crisis and emergency risk messages. Several reasons were identified, including not liking a particular spokesperson, having different value systems, lacking trust in the authority, being in disbelief that they might be at risk, or having faith in their ability to be self-sufficient in times of crisis. Littlefield (2013) added that "lack of resources, vulnerable living conditions, and possibly misguided or misunderstood communication efforts put underrepresented or vulnerable populations at greater risk during a crisis situation" (p. 2). Because minority or vulnerable publics may have different cultural influences that impact the way they receive and act upon risk and crisis messages, the present study sought to determine if these groups would perceive particular risk and crisis messages to be more effective than status quo messages and self-report their behavioral intentions to respond as directed by the crisis spokespeople.

\subsection{Idea Learning Cycle Model}

Previous research on instructional crisis messages has focused on stage two (information and explanation) of the four-stage cycle developed by Kolb (1984) to articulate how learning is achieved through concrete experience (feeling), reflective observation (watching), abstract conceptualization (thinking), and active experimentation (doing) (Frisby, Sellnow, Sellnow, Lane, \& Veil, 2011; Sellnow \& Sellnow, 2010). In addition, learning style preference does influence receiver interpretation, self-efficacy, and behavioral intention of instructional crisis messages (Giuse, Koonce, Storrow, Kusnoor, \& Ye, 2012; Sellnow, Sellnow, Lane, \& Littlefield, 2012b). Finally, instructional messages that include elements of "explanation, internalization, and action" result in significantly higher degrees of self-efficacy and behavioral intention to take appropriate action than those focused only on information and explanation (Sellnow \& Sellnow, 2010; Sellnow, Sellnow, \& Seeger, 2012a).

These studies provided the basis for the IDEA learning cycle comprised of internalization (I), distribution (D), explanation (E), and action (A) (D. Sellnow \& T. Sellnow, 2014; T. Sellnow \& D. Sellnow 2010, 2013). What remains uncertain is how messages that include all elements of the IDEA learning cycle model might differentially influence the interpretation of and response to crisis messages based on individual differences, including culture, sex, and socio-economic status. D. Sellnow et al. (2013) found that instructional messages addressing all elements of the IDEA model were perceived as more effective for the general population. However, when determining if these IDEA messages would have a greater impact on self-reported behavioral intentions than status quo messages, the findings were mixed. Furthermore, they did not address minority or ethnic publics in their study. Thus, the present study seeks to test the following hypotheses and research questions:

H1: Cultural groups will perceive instructional crisis messages that address all elements of the IDEA learning cycle model as more effective than status quo messages.

H2: Instructional crisis messages that address all elements of the IDEA learning cycle model will have a more positive impact on cultural groups' self-reported behavioral intentions than status quo messages.

RQ1: (a) To what degree does cultural groups' learning style preference affect message effectiveness?

(b) To what degree do cultural groups' behavioral intentions reflect the effectiveness of instructional crisis communication messages?

RQ2: What relationships exist among sex, learning style preference, and message type for cultural groups relative to message effectiveness and behavioral intentions? 


\section{Methods}

An experimental design using pre- and post-treatment measures was employed to test the hypotheses and research questions. Sampling procedures, participants, and instrumentation are provided below.

\subsection{Sampling Procedures}

The sample consists of participants drawn from survey site locations across the United States. Survey administrators made an effort to balance participants among men and women. Individuals were recruited from a large southeastern university, a community from the Deep South, church groups in the eastern U.S., a Native American community located in the upper Midwest, and a community college and university in the East-Central U.S. For the sample drawn from the large southern university, students were recruited from communication courses, and received class-assignment credit for completing the survey. All other study participants were paid twenty-five dollars for participating in the study. With the exception of the sample drawn from the Eastern U.S., all participants completed the online surveys at computer labs housed within institutions of higher education. Participants recruited from the Eastern U.S. completed the survey on computers at church community centers.

While the sample from the southern university was drawn from a communication course, the other participants were identified using non-random network sampling, where local cultural agents served as recruiters in the targeted communities. For example, the cultural agent in the upper Midwest community contacted local leaders and opinion leaders to spread the word about the survey and identify individuals who might be interested in participating. Individuals signed up to participate. The cultural agent in the Deep South recruited participants from the local community, many of whom were students. The cultural agent for the Eastern U.S. sample contacted church pastors, and a snowball method was used to recruit participants from churches in two communities. The pastors invited congregation members to take part in the study, and individuals signed up to participate. For the East Central U.S. sample, a cultural agent coordinated with faculty members of the community college and the university, and a snowball method using face-to-face and E-mail was used to recruit students for the study. Individuals signed up to participate.

Participants from all survey samples reported to locations where they were given instructions about using a laptop and headphone equipment to watch a stimulus message and respond to an online survey. Upon receiving instructions, interested individuals were provided access to the online survey where they consented to participate in the study. As participants accessed the online survey through Qualtrics.com, the survey system automatically assigned individuals to either a treatment or comparison group. Treatment group participants were assigned to watch a stimulus message that differed from the message presented to comparison group participants. The stimulus messages for each group consisted of a news clip and were approximately two-minutes in length. After watching the stimulus message, participants completed a survey, were debriefed and informed that the news clip was not real, and were excused from the lab.

\subsection{Participants}

The sample consisted of 746 participants with $49.6 \%(n=370)$ assigned to the treatment condition, and $50.4 \%$ $(n=376)$ assigned to the comparison condition. Of the participants, $39.4 \%(\mathrm{n}=294)$ were male. Additionally, $25.3 \%$ $(\mathrm{n}=185)$ of the participants identified themselves as African American Black, and $11.2 \%(\mathrm{n}=82)$ identified themselves as Native American. Furthermore, 40.3\% ( $\mathrm{n}=300)$ of the participants reported themselves as earning a low annual income, $12.9 \%(\mathrm{n}=96)$ a low-middle income, $28.3 \%(\mathrm{n}=211)$ a middle income, or $18.5 \%(\mathrm{n}=138)$ an upper-middle to high annual income. Of the participants, $25.5 \%(\mathrm{n}=190)$ have completed some schooling or have received a high school diploma, $15.5 \%(\mathrm{n}=115)$ have earned a two-year degree, and 59\% $(\mathrm{n}=439)$ have completed degrees at the Bachelor's, Master's, or Doctorate level. Finally, $14.2 \%(\mathrm{n}=106)$ reported being raised in a rural setting, 26.8\% $(\mathrm{n}=200)$ in a small town with a population between 5,000 and $25,000,30 \%(\mathrm{n}=224)$ in a mid-size city between 25,000 and $100,000,19.2 \%(\mathrm{n}=143)$ in a large city with a population between 100,000 to 500,000 , and $9.8 \%$ $(\mathrm{n}=73)$ in a major metro area. 
Table 1. Percentage of ethnicity of three largest categories by location and condition

\begin{tabular}{lllllll}
\hline Location & Condition & $\begin{array}{l}\text { African } \\
\text { American } \\
\text { or Black } \\
(\mathrm{n}=185)\end{array}$ & $\begin{array}{l}\text { Caucasian } \\
\text { White } \\
(\mathrm{n}=399)\end{array}$ & $\begin{array}{l}\text { or } \\
\text { Amative } \\
(\mathrm{n}=82)\end{array}$ & $\begin{array}{l}\text { Total } \\
(\mathrm{n}=666)\end{array}$ \\
\hline Southern U.S. & Control Message & 3.9 & 29.6 & 0.1 & 33.6 \\
& Treatment Message & 3.3 & 28.7 & 0.3 & 32.3 \\
\hline East Central U.S. & Control Message & 3.2 & 0.1 & 0.5 & 3.8 \\
& Treatment Message & 3.0 & 0.0 & 0.5 & 3.5 \\
\hline Eastern U.S. & Control Messag & 6.9 & 0.3 & 0.0 & 7.2 \\
& Treatment Message & 7.2 & 0.1 & 0.1 & 7.4 \\
\hline Upper-Midwest & Control Message & 0.3 & 0.9 & 5.0 & 6.2 \\
& Treatment Message & 0.0 & 0.1 & 5.9 & 6.0 \\
\hline Total & & 27.8 & 59.8 & 12.4 & 100.0 \\
\hline
\end{tabular}

\subsection{Message Stimulus "News Clips"}

For this study, two news clip stimulus messages were developed based on news scripts used to report a $2011 \mathrm{E}$. coli outbreak in ground beef in several New England states. For the comparison (a.k.a. control) condition message, the script mirrored the format of an actual news report. In contrast, the treatment (a.k.a. IDEA) condition message was similar in design but manipulated to include elements beyond information and explanation. More specifically, the IDEA message included elements of internalization and action. Food scientist experts from the National Center for Food Protection and Defense provided input for the message development to make sure the claims being made were scientifically sound. Additionally, broadcast news experts provided input to make sure that the message content and format of reports aligned with industry standards. With the inclusion of expert input, the messages were edited to be similar in length (approximately 500 words each). To make the messages appear realistic as viewed by survey participants, broadcast news industry professionals were hired to record the messages.

\subsection{Instrumentation}

\subsubsection{Learning Style Quiz (LSQ)}

The LSQ (Sellnow, 2005) is a 15-item scale asking participants to select from four nominal level learning style descriptors the one they perceive to be most like them. Responses to the fifteen items are first summed based on a preference toward feeling (concrete experience), watching (reflective observation), thinking (abstract conceptualization), or doing (active experimentation). These sums are then combined systematically (i.e., feeling + watching $=$ stage 1 preference; watching + thinking $=$ stage 2 preference; thinking + doing $=$ stage 3 preference; doing + feeling $=$ stage 4 preference) to determine a learning preference for each participant. The category with the highest value is assigned as the preferred learning style for each participant. For example, a participant whose summative scores are 4 (stage 1), 8 (stage 2), 7 (stage 3), and 1 (stage 4) would be coded as having a stage 2 learning style preference (Venette, 2014).

In the data set for this study, only participants with a distinct (high score) preference were included in the analyses. Data were discarded when ties occurred (e.g., 3, 8, 8, 1 - indicating an equal learning style preference for both stages 3 and 4). Thus, $20.9 \%(n=156)$ of the participants were not included in the analyses. In terms of usable data, $31.2 \%$ ( $n=233)$ were coded as Divergers with a stage 1 learning style preference (i.e., feeling + watching), 19.2\% ( $n=143)$ as Assimilators with a stage 2 learning style preference (i.e., watching + thinking), $14.1 \%(n=105)$ as Convergers with a stage 3 learning style preference (thinking + doing), and 35.5\% ( $\mathrm{n}=265)$ as Accommodators with a stage 4 learning style preference (i.e., doing + feeling).

\subsubsection{Perceived Message Effectiveness}

Seven items were adapted from Harris (2007) and Noar, Palmgreen, Zimmerman, Lustria, \& Lu, (2010) to measure perceived message effectiveness. Items included, "this video would catch my attention," "this video would make me more likely to not eat potentially contaminated food," "this video is memorable," "this video is effective," "this 
video would make people my age more likely to not eat potentially contaminated food," "this video would help convince people my age to not eat potentially contaminated food," and "this video would help convince me to not eat potentially contaminated food." All items were measured using a 5-point Likert scale ranging from strongly disagree (1) to strongly agree (5). The one-dimensional, seven-item scale was reliable $(\alpha=.88, M=26.50, S D=4.96)$.

\subsubsection{Behavioral Intentions}

Two of the 12 individual items included in the instrument were used to measure specific behavioral intentions associated with food contamination. Items included: "based on the video you just saw, how likely would you be to return your ground beef to the store where you purchased it," "how likely would you be to ask someone else what they would do?"

\section{Results}

The first hypothesis predicted that cultural groups will perceive instructional crisis messages that address all elements of the IDEA learning cycle model as more effective than status quo messages. Hypothesis 1 was tested by calculating a 2 (condition) x 3 (ethnicity) univariate analysis of variance. Overall results revealed significant main effects for both condition and ethnicity. Participants who viewed the treatment message $(\mathrm{M}=3.94$, s.d. $=.75)$ reported that the message was more effective than participants who viewed the comparison message $(\mathrm{M}=3.88$, s.d. $=.75)[\mathrm{F}(1,664)=3.28, \mathrm{p}<.05]$. African American participants $(\mathrm{M}=4.13$, s.d. $=.73)$ and Native American participants $(\mathrm{M}=4.05$, s.d. $=.76)$ reported that the messages they viewed were more effective than Caucasian participants $(\mathrm{M}=3.78$, s.d. $=.72)[\mathrm{F}(2,664)=15.98, \mathrm{p}<.01]$. While Caucasian participants reported lower levels of message effectiveness than African Americans or Native American participants, results revealed no significant interaction effects of condition by ethnicity on message effectiveness $[\mathrm{F}(2,664)=2.17, \mathrm{p}>.05]$. Thus, Hypothesis 1 was partially supported.

Table 2. Descriptive statistics for main effects for both condition and ethnicity

\begin{tabular}{lllll}
\hline Ethnicity & Condition & Mean & Std. Deviation & N \\
\hline African Americans or Black & Control Message & 4.13 & .72 & 94 \\
& Treatment Message & 4.12 & .75 & 89 \\
\hline Caucasian or White & Control Message & 3.76 & .71 & 206 \\
& Treatment Message & 3.9 & .73 & 193 \\
\hline Native Americans & Control Messag & 3.85 & .83 & 37 \\
& Treatment Message & 4.21 & .66 & 45 \\
\hline Total & Control Message & 3.88 & .75 & 337 \\
& Treatment Message & 3.94 & .75 & 327 \\
\hline
\end{tabular}

The second hypothesis predicted that instructional crisis messages that address all elements of the IDEA learning cycle model will have a more positive impact on cultural groups' self-reported behavioral intentions than status quo messages. A one-way analysis of variance (ANOVA) revealed that individuals who saw the treatment message were more likely to return the ground beef to a store $[F(1,745)=21.87, \mathrm{p}<.0001]$ and less likely to ask someone else what to do $[F(1,744)=3.52, \mathrm{p}<.05)$. As such, the second hypothesis was supported.

Research Question 1 asked “(a) To what degree does cultural groups' learning style preference affect message effectiveness and (b) behavioral intentions in response to instructional crisis communication messages?" To examine RQ1(a), a series of 2 (condition) x 3 (ethnicity) x 4 (Learning Style Preference) univariate analyses were conducted to explore whether learning style preference affects message effectiveness and behavioral intentions in response to instructional crisis communication messages. Results indicated a main effect for ethnicity. Caucasian participants reported significantly lower levels of message effectiveness than either Native Americans or African Americans $[F$ $(2,664)=13.32, \mathrm{p}<.0001]$. Results did not reveal a significant interaction between condition and ethnicity. For example, Native Americans who saw the control message reported lower levels of message effectiveness than did Native Americans in the treatment condition $(\mathrm{p}>.05)$.

To examine RQ1(b), a series of 2 (condition) x 3 (ethnicity) x 4 (LSQ) univariate analyses were conducted to explore whether learning style preference affects behavioral intentions in response to instructional messages. Results indicated a significant main effect for condition and ethnicity when reviewing the intent of individuals to return the 
ground beef to the store. Individuals who saw the treatment message were more likely to return the ground beef as recommended $[F(1,665)=4.61, \mathrm{p}<.05]$. Regardless of condition, African Americans reported significantly greater likelihood of returning the ground beef than either Native Americans or Caucasians $[F(2,664)=9.19, \mathrm{p}<.0001]$. When reviewing the intent of individuals to ask someone else what to do, results did not reveal significant results or main effects.

Research Question 2 asked, "What relationships exist among sex, learning style preference, and message type for cultural groups relative to message effectiveness and behavioral intentions?" To examine RQ2, a series of 2 (condition) x 3 (ethnicity) x 4 (LSQ) x 2 (sex) multivariate analysis of variance (MANOVA) were conducted to explore what relationships exist between message type, ethnicity, and learning style preference relative to message effectiveness and behavioral intentions. Significant four-way interaction was found $[F(6,663)=2.18, \mathrm{p}<.05]$. Native American and African American females in the treatment group who report a LSQ preference of converger were more likely to return the ground beef to a store, than any other group.

\section{Discussion}

The findings suggest variability between ethnic groups and whites regarding receptivity to risk and crisis messages providing support for continued study moving beyond the sender-oriented approach and focusing on the receiver-oriented view of message creation and presentation. As D. Sellnow et al. (2013) suggested, best practices are central to effective message preparation and distribution for all audiences. However, risk and crisis messages may be more effective with particular groups if more attention is focused on particular cultural groups to whom the messages are intended.

The data from the present study supported Hypothesis 1, extending prior scholarship suggesting that risk and crisis messages incorporating the IDEA learning cycle would be perceived as more effective by the publics than messages focusing only on information about the crisis (Sellnow et al., 2012b; D. Sellnow et al., 2013). Participants viewing the IDEA message perceived it as significantly more effective than the traditional clip. Furthermore, African American and American Indian participants viewed the messages as significantly more effective than Caucasian participants. There are several reasons why this may have been the case. First, previous research suggests that a high percentage of minority populations get more of the news from media and tend to believe what they view as the truth (Guskin, Moore, \& Mitchell, 2011). In addition, the IDEA learning cycle includes personally relevant information that resonates with groups that are more collectively oriented. Studies suggest that African Americans and American Indians are collectivistic in caring for members of their communities (Triandis \& Triandis, 1990).

With regard to Hypothesis 2, the IDEA messages significantly motivated participants to indicate their behavioral intent to return the contaminated beef to the store. However, the findings did not reveal a significant difference between groups when asked about indicating if they would seek information from others about what to do. There may be reasons for this associated with individualism and self-efficacy. Once personally connected to the issue, informed about the risk, and instructed what to do, individuals appeared motivated to take action and return the beef. By asking another for information or assistance, the individual would have demonstrated dependence.

For Research Question 1, results suggest that ethnicity made a difference in some cases regarding perception of message effectiveness and behavioral intention. African Americans and American Indians perceived the messages as more effective than did the Caucasian participants. African Americans also reported a significantly greater likelihood of returning the ground beef. Clearly, the impact of the message may have appeared to be influenced by the ethnicity of the receiver. For example, the treatment messages used individuals of American Indian or African American heritage as examples of victims of the contamination. Identifying with someone having a similar origin may have influenced the viewer to pay more attention to the message. This supports the notion that spheres of ethnocentricity (Littlefield, 2013) - identified as a focus on self or those closest to self as most relevant - may have had a strong impact on those appearing to be most directly affected by the crisis. Because the media were tailored to include representatives from the particular ethnic groups as victims of the contamination, Caucasian viewers did not see themselves as victims. The composition of the media may have contributed to the receptivity of the ethnic participants to the message.

Finally, Research Question 2 added sex to the mix with the results suggesting that American Indian and African American women who reported a learning style preference of converger were significantly more likely to return the ground beef to the store. Previous research suggests that women are the primary food preparers (Hale, 2011), are likely to be the ones who purchase food, get information about food sources, and learn about potential problems associated with food preparation (Sellnow et al., 2012b). 
Future research should continue exploring a range of audience variables and matching them with congruent message strategies. D. Sellnow et al. (2013) suggested that organizations with retail activity geared toward one sex or another might focus their messages toward strategies that are preferred by that sex. Conversely, if a strategy has little impact on an audience, that strategy need not be included. More research is needed.

This study is limited in several ways. First, the audience measures are based on a simulation. Newscasts were designed to be as authentic as possible, but those who viewed and responded to them did so in a variety of conditions that may not have been as conducive to gaining a favorable response. Second, although the messages were similar in length, they were somewhat longer than a typical news report. Thus, length of stimulus messages may have encouraged viewer fatigue, disinterest, or distraction, which in turn may have negatively influenced thoughtful responses. Third, the study used measures of self-reporting that did not measure actual behavior. Fourth, the study involved a single measurement period. Doing so did not allow for any shifting of opinion over time. Finally, the study was limited to one crisis type. Responses may differ in crisis forms other than a food contamination.

Crisis situations are fraught with uncertainty. As such, one goal for crisis communicators is to reduce that uncertainty for all groups through messages intended to provide instructions for how to mitigate or prevent a crisis from occurring. The focus of this research was to ascertain if particular cultural groups perceived and/or responded to risk and crisis messages differently than the general population. As the present study suggested, not all groups perceived and/or responded to crisis messages similarly. Thus, these findings provide justification for continued research into receiver-based crisis communication, particularly with diverse cultural groups in mind, if we are to move closer to the goal of providing crisis messages that will help those affected by crises to mitigate or prevent them from happening.

\section{References}

Andrulis, D. P., \& Brach, C. (2007). Integrating literacy, culture, and language to improve health care quality for diverse populations. American Journal of Health Behavior, 31, S122-133. http://dx.doi.org/10.5993/AJHB.31.s1.16

Frisby, B. N., Sellnow, D. D., Sellnow, T. L., Lane, D. R., \& Veil, S. R. (2011). Instructional messages in times of crisis: Targeting learning preferences and self-efficacy. Paper presented at the International Communication Association Conference, Boston, Massachusetts.

Giuse, N. B., Koonce, T. Y., Storrow, A. B., Kusnoor, S. V., \& Ye, F. (2012). Using health literacy and learning style preferences to optimize the delivery of health information. Journal of Health Communication, 17, 122-140. http://dx.doi.org/10.1080/10810730.2012.712610

Guskin, E., Moore, P., \& Mitchell, A. (2011). The state of the news media: An annual report on American journalism. Pew Research Center's Project for Excellence in Journalism. Retrieved from $\mathrm{http}: / /$ stateofthemedia.org/2011/african-american/

Hale, T. (2011, March 11). In U. S. men are shopping more than ever, while women are watching more TV. Neilson Wire. Retrieved from http://blog.nielsen.com/nielsenwire/consumer/in-u-s-men-are-shopping-more-than-ever-while-women-are-watc hing-more-tv/

Harris, M. S. (2007). The role of emotion in anti-drug PSA: Investigating the impact of guilt arousal on perceived message effectiveness and behavioral intentions to use drugs. Dissertation Abstracts International Section A: Humanities and Social Sciences, 68(4a), 1202.

Kolb, D. A. (1984). Experiential learning: Experience as the source of learning and development. Englewood Cliffs, NJ: Prentice Hall, Inc..

Kreps, G. L., Alibek, K., Bailey, C., Neuhauser, L., Rowan, K. E., \& Sparks, L. (2005). The critical role of communication to prepare for biological threats: Prevention, mobilization, and response. In J. A. Johnson, G. R. Ledlow, M. A. Cwiek (Series Eds.), \& D. O’Hair, R. L. Heath, \& G. A. Lewlow (Vol. Eds.). Community preparedness and response to terrorism. Volume 3: Communication and the media (pp. 191-210). Westport, CT. Praeger.

Lindell, M. K., \& Perry, R. W. (2004). Communicating environmental risk in multiethnic communities. Thousand Oaks, CA: Sage. 
Littlefield, R. S. (2013). Communicating risk and crisis communication to multiple publics. In A. J. DuBrin (Ed.), Crisis leadership in organizations (pp. 231-251). Northampton, MA: Edward Elgar Publishing Ltd. http://dx.doi.org/10.4337/9781781006405.00021

Noar, S. M., Palmgreen, P., Zimmerman, R. S., Lustria, M. L. A., \& Lu, H. Y. (2010). Assessing the relationship between perceived message sensation value and perceived message effectiveness: Analysis of PSAs from an effective campaign. Communication Studies, 61(1), 21-45. http://dx.doi.org/10.1080/10510970903396477

Sarbaugh, L. E. (1979). Intercultural communication. Rochelle Park, NJ: Hayden Book Company, Inc.

Seeger, M. W., Sellnow, T. L., \& Ulmer, R. R. (2003). Communication and organizational crisis. Westport, CN: Praeger.

Sellnow, D. D. (2005). Confident public speaking ( $2^{\text {nd }}$ ed.). Boston, MA: Cengage.

Sellnow, D. D., \& Sellnow, T. L. (2014). Instructional principles, Risk Communication. In T. L. Thompson (Ed.), Encyclopedia of health communication (pp. 1181-1182). Thousand Oaks, CA: Sage.

Sellnow, D. D., Lane, D. R., Littlefield, R. S., Sellnow, T. L., Beauchamp, K. A., \& Wilson, B. (2013). An individual differences receiver-based examination of instructional crisis communication. Paper accepted for presentation at the International Communication Association Convention, London, England.

Sellnow, T. L., \& Sellnow, D. D. (2010). The instructional dynamic of risk and crisis communication: Distinguishing instructional messages from dialogue. The Review of Communication, 10(2), 111-125. http://dx.doi.org/10.1080/15358590903402200

Sellnow, T. L., Sellnow, D. D., \& Seeger, M. W. (2012a, October 26). Theorizing organizational crisis communication: Challenges and opportunities. Paper presented at the European Communication Research and Education Association Conference, Istanbul, Turkey.

Sellnow, T. L., Sellnow, D. D., Lane, D. R., \& Littlefield, R. S. (2012b). The value of instructional communication in crisis situations: Restoring order to chaos. Risk Analysis, 34(4), 633-643. http://dx.doi.org/10.1111/j.1539-6924.2011.01634.x

Sellnow, T. L., Ulmer, R. R., Seeger, M. W., \& Littlefield, R. S. (2009). Effective risk communication: A message-centered approach. New York, NY: Springer. http://dx.doi.org/10.1007/978-0-387-79727-4

Sellnow, T., \& Sellnow, D. (2013, July). The role of instructional risk messages in communicating about food safety. Food Insight: Current Topics in Food Safety and Nutrition, International Food Information Council, 3.

Spence, P. R., Lachlan, K. A., \& Griffin, D. R. (2007). Crisis communication, race, and natural disasters. Journal of Black Studies, 37(4), 539-554. http://dx.doi.org/10.1177/0021934706296192

Triandis, H. C., \& Triandis, J. B. (1990). Cross-cultural studies of individualism and collectivism. In J. Berman (Ed.), Nebraska symposium on motivation, 1989 (pp. 41-133). Lincoln, NE: University of Nebraska Press.

Venette, S. J. (2014). Venette Learning Style Calculator (Version 2) [Software]. 